Recepción: 09 / 02 / 2018

Aceptación: 18 / 04 / 2018

Publicación: 01 / 08 / 2018
Ciencias sociales y políticas

Artículo de investigación

\title{
Dinámicas migratorias: México y ciudad de Juárez \\ Entre historias de vida y tensiones
}

Migratory dynamics: México and Ciudad de Juárez.

Between life stories and tensions

Dinâmica migratória: México e Ciudad de Juárez

Entre histórias de vida e tensões

\author{
Mauricio Muñoz-Landázuri ${ }^{\mathrm{I}}$ \\ mauriciomunoz_96@hotmail.com \\ Maricela Sánchez-Morante II \\ marysan1976@hotmail.com \\ Carla Barberán-Cornejo III \\ carbabarberan99@gmail.com \\ Roberth Párraga-Cepeda IV \\ roberthcepeda@hotmail.com
}

Correspondencia: mauriciomunoz_96@hotmail.com

\footnotetext{
IEstudiante de la Escuela de Sociología y Ciencias Políticas de la Universidad de Guayaquil, Guayaquil, Ecuador.

II Magister en Educación Superior, Docente de la Universidad de Guayaquil, Guayaquil, Ecuador.

III Estudiante de Medicina de la Universidad Católica de Santiago de Guayaquil, Guayaquil, Ecuador.

${ }^{\text {IV }}$ Magister en Docencia Universitaria e Investigación, Docente de la Universidad de Guayaquil, Guayaquil, Ecuador.
} 


\title{
Resumen
}

La migración tiene una importancia geo-referencial porque incluye distintos procesos y actores sociales que marcan la vida de las ciudades involucradas en esta dinámica social. Esta investigación tiene como objetivo visibilizar el fenómeno migratorio en el caso específico de Ciudad Juárez, situado en la frontera norte de México. El siguiente trabajo analiza las perspectivas y experiencias de las personas que han vivido la migración desde diferentes roles: agentes migratorios americanos, migrantes ilegales deportados y polleros, quienes organizan el traslado ilegal a Estados Unidos.

Palabras claves: migración; migrantes ilegales; agentes migratorios; polleros; Ciudad Juárez.

\begin{abstract}
Migration has a referential geo important because it includes different processes and social actors that makes the life of the cities involved in this social dynamic. This research aims to visualize the migratory phenomenon in the specific case of Ciudad Juarez, located on the northern border of Mexico. The following paper analyzes the perspectives and experiences of people who have experienced migration from different roles: American immigration agents, deported illegal migrants and polleros who organized the illegal transfer to the United States.
\end{abstract}

Keywords: migration; illegal migrants; immigration agents; polleros; ciudad Juarez.

\section{Resumo}

A migração tem um geo referencial importante porque inclui diferentes processos e atores sociais que tornam a vida das cidades envolvidas nessa dinâmica social. Esta pesquisa visa visualizar o fenômeno migratório no caso específico de Ciudad Juarez, localizado na fronteira norte do México. O artigo a seguir analisa as perspectivas e as experiências de pessoas que vivenciaram a migração de diferentes funções: agentes de imigração americanos, deportados migrantes ilegais e polleros que organizaram a transferência ilegal para os Estados Unidos.

Palavras chave: migração; migrantes ilegais; agentes de imigração; polleros; ciudad Juarez. 


\section{Introducción}

Es en este punto en el que radica la propuesta de nuestra investigación, de las entrevistas hechas a migrantes, polleros y agentes migratorios se rescatará la perspectiva humanitaria que gira en torno al fenómeno migratorio, buscando romper los estigmas, estereotipos y prejuicios que se tiene sobre quienes participan en dicho fenómeno: como el aseverar que tanto agentes migratorios como polleros le dan mal trato a quienes buscan emigrar de manera no legal. Así mismo se busca evitar que al momento de observar el flujo migratorio se haga únicamente desde una perspectiva centrada en los datos duros. Se propone rescatar el interés en las cuestiones sensibilizadoras que atraviesan dichos datos.

\section{Antecedentes}

Desde el principio de los tiempos el ser humano se ha visto envuelto en la dinámica de la migración, recordemos, por ejemplo, aquellos antepasados nómadas, que se trasladaban de un lugar a otro buscando satisfacer sus necesidades, principalmente de alimentación y de seguridad. Hoy en día, la reproducción de dicha práctica no siempre es la opción más sencilla para las personas que deciden salir de su país de origen ya sea por necesidades económicas, de seguridad, o por mero gusto y deseo de sobresalir, puesto que con el pasar de los años se van creando y reformulando políticas, estrategias e iniciativas con miras a controlar el flujo migratorio. La insistencia de éstas por parte de los países involucrados puede ser más fácilmente entendible si tomamos en cuenta los impactos que se le atribuyen, como cuestiones económicas y sociales, entre otras, además de que las consecuencias que traen consigo se suponen mayormente negativas, tanto para el país de origen como para el país de destino de los y las migrantes (en algunos casos las consecuencias abarcan también a los países situados entre el de origen y el de destino, como es el caso de México en relación a los y las migrantes de centro y Sudamérica).

Sobre los impactos de la migración en lo social, Mazurek hace mención de "la desestructuración familiar, de los sistemas comunitarios y de las culturas locales (en países donde las formas tradicionales de cultura predominan)" (Mazurek, 2009:22), aspectos que preocupa que sean dañados dentro de un país, especialmente, si se tiene la idea de que son estos mismos los que dotan de identidad y coerción social al mismo. 
Este mismo autor habla acerca del impacto territorial que gira en torno al proceso migratorio: “existe un impacto territorial que se materializa por la recomposición de la fuerza de trabajo a escala mundial y continental, pero también a nivel local” (Mazurek, 2009:23). Dicho impacto territorial nos remite a la vez al desplazamiento de las zonas rurales por cuestiones globalizadoras (retomando las ideas de Jeremy Rifkin), que haciendo a un lado al campesinado se le obliga a migrar a donde sea posible, ya sea dentro de su Estado nación, o hacia el extranjero, con tal de encontrar una forma de vida donde encajen los conocimientos que han adquirido con el paso del tiempo. Vale mencionar que estas analogías nos remontan en términos históricos en lo que a México y Estados Unidos se refiere. El primero de ellos fue el Programa Bracero, que fue implementado posterior a la Segunda Guerra Mundial, y el siguiente fue la crisis de los Estados Unidos en el periodo de 1942-1964. Con este programa millones de mexicanos dejaron sus tierras, en su mayoría campesinos, tierras que gradualmente quedaron despobladas. Al concluir el programa bracero, 22 años después vuelven los trabajadores, ha pasado aproximadamente una generación de jóvenes, generación que al volver se encuentran con otro momento histórico: el inicio de la Industria Maquiladora en México, que dio inicio con la intención de cubrir la necesidad de empleo que se originaría en México, agravado por la situación del regreso de los ahora ex braceros.

Si bien es cierto que la migración no es un fenómeno reciente, también es cierto que no siempre ha sido vista como totalmente negativa para los países involucrados, así mismo, las políticas creadas para moderar el flujo migratorio no presumen de una constante rigurosidad a la hora de ser llevadas a la práctica.

Pues bien, el origen de la migración permanente de México a Estados Unidos se sitúa, según Rodríguez Ramírez, a finales del siglo antepasado "cuando en el suroeste de aquel país se emprendieron grandes obras de infraestructura encabezadas por la construcción del ferrocarril, las presas y los sistemas de riego" (Rodríguez Ramírez, 2007:29), a partir de entonces, las políticas en Estados Unidos sobre temas de migración "han sido muy sensibles a los vaivenes de la economía" (Padilla, 2012:215), lo cual se constata al repasar los datos históricos de esta frontera, en que los flujos migratorios se potenciaron en contextos de la Revolución mexicana y la Primera Guerra mundial, así como sucedió a la inversa, cuando el flujo migratorio incrementó de Estados 
Unidos a México durante la crisis económica de Norteamérica por causa de las repatriaciones masivas llevadas a cabo alrededor de 1930 (Rodríguez Ramírez, 2007; Padilla, 2012).

La inconsistencia referente a la regulación de la migración a través de su historia entre México y Estados Unidos, ha ido de la mano con la inconsistencia del pensamiento social sobre este tema, que, además, cabe decir, es diferente en cada uno de los países involucrados en el fenómeno migratorio. Una de las posibles causas de lo anterior es lo que menciona Burbano Alarcón (2010) cuando habla de la influencia de los medios de comunicación sobre los prejuicios e ideas que se forma la sociedad entorno a los y las migrantes, pues "culpabilizar a los migrantes puede servir para justificar problemas económicos, sociales o políticos de una sociedad" (Burbano Alarcón, 2010: 176), por lo cual se puede deducir que en los momentos en que sea necesario, se podría tomar la imagen de los y las migrantes como chivos expiatorios en los que, sin tener que ser cierto, se descargue la responsabilidad de cualquier clase de problemas que invadan la nación a la que han llegado de manera ilegal.

\section{Objetivos de la Investigación:}

\section{Objetivo General:}

Analizar el fenómeno migratorio de Ciudad Juárez, desde las perspectivas y experiencias de las personas que han vivido la migración desde los diferentes roles: agentes migratorios americanos, migrantes ilegales deportados y polleros.

\section{Objetivos Específicos:}

Reconocer la importancia del fenómeno migratorio que tiene lugar en Ciudad Juárez.

Distinguir las vivencias positivas y negativas que han experimentado migrantes, agentes migratorios y polleros en torno a la migración.

Diagnosticar dichas vivencias desde una perspectiva teórica de estigmas sociales, estereotipos y prejuicios.

\section{Justificación}

La migración internacional es un fenómeno que ha llamado la atención tanto de investigadores como de aquellos que toman decisiones entorno a las políticas y leyes que la regulan, teniendo 
como fin desde entender el fenómeno migratorio en cada uno de los aspectos que lo conforman, hasta el buscar las soluciones necesarias al "problema".

Gracias a dicho interés es que actualmente encontramos datos duros (generalmente en lapsos de tiempo) sobre las personas que se trasladan de un país a otro, tanto de manera legal como de manera ilegal, es así como podemos afirmar que, según el Reporte Ejecutivo de la Secretaría de Relaciones Exteriores (SRE), “en 2004, había aproximadamente 11.2 millones de mexicanos en los Estados Unidos" de los cuales "5.3 millones tenían un status legal y los otros 5.9 millones eran indocumentados" (Escobar, Martin, Cords. 2013), datos que no se pueden dejar de lado, pues nos hablan de la trascendencia de la migración de México a Estados Unidos, y nos ayudan a confirmar que es un asunto vigente.

Sin embargo, al momento de indagar en la información estadística es pertinente recordar que del total de esas cifras, cada unidad hace referencia a una persona que, al igual que cualquier otra dentro de un margen social, tiene familia, amigos, amigas, ambiciones, deseos de prosperar, sentimientos y pensamientos; los cuales le acompañan (aunque en ocasiones sea solo en el recuerdo) desde el momento de tomar la decisión de salir de su país de origen, y con los que atraviesa cada situación y experiencia -buenas y malas- durante su proceso de migración (en el caso de migrantes), así como lo hacen de la misma manera quienes emprenden cada amanecer su jornada laboral como agentes migratorios, enfrentándose a los peligros que conlleva dicha profesión, y finalmente, como sucede también con aquellos que consideran hacen un servicio a la comunidad cruzando a migrantes sin documentos para ayudarles a realizar su sueño americano.

\section{Estado de la cuestión}

En muchas ocasiones tratar de explicar la realidad social de un fenómeno se torna un problema complejo dada la cantidad de factores y variables a las que es necesario recurrir para que la explicación tenga sentido y también por la diversidad de puntos de vista desde los que se puede observar el mismo hecho, convirtiéndose así en un estado de la cuestión, ya que un mismo fenómeno podría estar siendo observado desde distintos puntos de vista, varios enfoques o perspectivas, también un mismo fenómeno podría verse inmerso en otras problemáticas. Dado por entendido lo anterior podemos aceptar que existen infinidad de estudios sobre el caso migratorio, sin embargo, cada uno de los estudios ya realizados difieren en cuestiones de 
enfoque, alcance y dirección de la investigación, dándole así importancia a cada uno de ellos, aunque parezca ya una temática acabada.

En la revisión de la literatura y los estudios previos a esta investigación, nos encontramos con una amplia diversidad de enfoques, por cuestiones de tiempo y espacio, pero si logramos resaltar los estudios más relevantes: Algunos de estos estudios presentan la perspectiva política que gira en torno al fenómeno de la migración ilegal, dentro de esta temática se encuentra la obra de Ana María López Sala (2005) «Inmigrantes y estados: la respuesta política ante la cuestión migratoria» en el cual afirma que la migración internacional es un problema, ya que llega a alterar las políticas de los Estados, tanto del que se emigra como al que se inmigra, ya sea por motivos de residencia o de empleo. Es por esto que "Las cuestiones planteadas aluden, en última instancia, a la significación que adquiere en las migraciones internacionales el establecimiento de fronteras políticas" (López Sala, 2005:14), mismas fronteras que suelen ser imaginarias y a su vez sancionadas legalmente.

Resaltamos también la obra de Richard R. Hofstetter (1990) «La política de inmigración de los Estados Unidos», que, mediante una serie de artículos elaborados por varios autores, muestra un panorama general de la situación de inmigración en los Estados Unidos, así como la necesidad de tener un control que estabilice o regule la población inmigrante en Estados Unidos, creando políticas para este fin, las cuales están en constante discusión dada la diversidad de puntos de vista y situaciones que ponen en juego a los Derechos Humanos Universales. Dicha dicotomía está plasmada en un artículo dentro de la obra mencionada «Política estadounidense de inmigración: ¿Cuán estrecha es la puerta?»- De Franklin S. Abrams en donde dice: “debe mencionarse que la mayoría de los inmigrantes se establece en los viejos centros urbanos, donde ya se han desarrollado infraestructuras. (...) Sin el ingreso de inmigrantes serían ahora grandes desiertos" (Abrams, 1990: 207).

\section{Marco teórico}

Para los fines de este artículo de investigación se discutirán los temas de estigma desde la teoría de Erving Goffman, esto situándonos en una visión sociológica, mientras que, por parte de la psicología (social) se propone la discusión conforme a temas de estereotipos y prejuicios, todo 
con el fin de tener una visión más amplia del proceso de migración y las perspectivas sociales que se crean en torno a sus actores sociales.

Comenzando con la obra de Erving Goffman (1963) «Estigma: La identidad deteriorada», y es que dentro de ésta se manejan varios tipos de estigmas (tres) que, en resumidas cuentas, se trata de «estigmas por cuestiones anatómicas o físicas, mentales y sociales», estas últimas siendo las que más nos interesan, acompañadas mínimamente por el estigma de cuestiones anatómicas o físicas, ya que como el autor las menciona se refieren a características bien definidas como lo son "las abominaciones del cuerpo -las distintas deformidades físicas- (...) Por último existen los estigmas tribales de la raza, la nación y la religión, susceptibles de ser transmitidos por herencia y contaminar por igual a todos los miembros de una familia." (Goffman, 1963:14).

Si bien en la dinámica de la migración no es muy discutido el tema de las deformaciones físicoanatómicas, la importancia de este estigma recae en un tema discutido durante muchos años entorno a la migración, como lo es la cuestión propia del fenotipo que, abusando del enfoque de Goffman, se trata de un estigma físico-anatómico ya que se hace una distinción y aislamiento por cuestiones de este tipo, como lo puede ser la estatura, el color de piel, el lenguaje, la nacionalidad, etc., en lo que se mezcla con el tercer estigma que está más relacionado con el aspecto social. La estigmatización no recae en la sociedad por idealización propia, puede ser por un grupo precursor, o bien por los medios de comunicación, ya que "los medios de comunicación, para bien o para mal, juegan un papel importante en la construcción de imaginarios sobre los inmigrantes y las sociedades receptoras" (OIM en Burbano Alarcón, 2010:171). De igual manera sobre los medios de comunicación se hace un señalamiento el cual se refiere a que los medios de comunicación hacen hincapié en los aspectos que segregan dejando efectos negativos al igual que canales o programas donde se resaltan los defectos de los migrantes o trabas que podrían llegar a provocar en la nación anfitriona. (Zapata-Barrero en Burbano Alarcón, 2009).

Referente a los temas de estereotipos y prejuicios, seguiremos, principalmente, lo escrito por Kassin, Fein y Markus (2010) en la $7^{\text {a }}$ edición de su libro <<Psicología Social $>>$. Pues bien, los estereotipos son definidos como la "creencia que asocia determinados rasgos o características a un grupo" (Kassin, et al. 2010:133), en cuya creación influyen varios factores, tales como la 
cultura y la cognición, la categorización social, y la pertenencia arraigada o no a determinados grupos, entre otros. En la cuestión de la cultura y la cognición, los autores hacen mención sobre la relevancia de los medios de comunicación para que las personas, como sociedad, estereotipemos a los grupos de aquellas y aquellos que son diferentes en alguna medida al grupo al que pertenecemos. Así "las películas y los programas televisivos de "realidad" que vemos, la publicidad a la que estamos expuestos, las bitácoras electrónicas (blogs) y los periódicos que leemos, ponen ante nuestros ojos imágenes estereotípicas y conceptos preestablecidos sobre los demás" (Kassin, et al 2010:134), este tema es retomado por Padilla (2012) cuando menciona que, algunas notas periodísticas sobre delitos cometidos en Estados Unidos, enfatizan en el lugar de origen del delincuente, o su situación de migrante ilegal,

Pero estas notas de los periódicos reproducen discursos alarmistas emitidos por autoridades, sin contribuir a un examen detenido sobre el posible impacto de la llegada de estas personas en la seguridad pública. Mucho menos, a la comprensión de las necesidades de atención que tienen estas personas. En este sentido, mientras lo primero cae en el terreno de la especulación, el problema tangible en relación con los deportados radica en las necesidades de atención inmediata: alojamiento, alimentación y atención médica, legal y psicológica a las personas (Padilla, 2012:219).

Un segundo factor (y el último que retomaremos para esta investigación, conforme al tema de estereotipos) que influye en la creación de estereotipos en las personas se denomina 'categorización social', la cual consta de agrupar a personas por características en común, aunque es un proceso natural de economía mental, también es necesario resaltar que al llevar a cabo la categorización social se suelen exagerar las diferencias entre los grupos, mientras que se minimizan las diferencias existentes dentro de cada grupo (Kassin, et al. 2010), un ejemplo vago de este tema con referencia a la migración, podría ser la categorización social de 'inmigrantes ilegales', categoría en la cual se minimizarían las diferencias de país de origen, motivo de migrar, lugar por el que se ingresó al país de destino, entre muchas otras cuestiones que contribuyen a que estas personas sean vistas y tratadas desde diferentes puntos. Así mismo, se podrían exagerar las diferencias existentes entre los y las inmigrantes ilegales, y quienes migraron de manera legal, o quienes son originarios de los Estados Unidos (en este análisis), las cuales pueden radicar exclusivamente en la obtención o no de papeles de residencia. 
Son varias las formas en las que se perpetúan los estereotipos, como son las correlaciones ilusorias "una tendencia de la gente a sobreestimar el vínculo existente entre variables que en realidad sólo están relacionadas ligeramente o no lo están en absoluto” (Kassin et al, 2010:138) para poner un ejemplo de esta última, digamos que una persona migrante tiene formado un estereotipo acerca de los agentes migratorios, que consiste en creer y aseverar que estos son personas groseras, agresivas, intolerantes, y que siempre tratan mal a cualquier persona que busca ingresar a su país sin documentos legales; este migrante, al momento de toparse con algún agente migratorio buscará (aunque no precisamente de manera consciente confirmar su estereotipo, aunque esto signifique minimizar o anular las actitudes positivas que él o la agente pudiera tener hacia sí, además de esto, el migrante estaría predispuesto con una actitud posiblemente a defensiva para comenzar a tratar el 'problema', lo que muy probablemente podría dar como consecuencia que él o la agente de migración actúe de manera que confirme el estereotipo del migrante.

Finalmente, un prejuicio se define como los "sentimientos negativos hacia otras personas con base en su filiación a ciertos grupos" (Kassin et al, 2010:133), aunque estos tienen influencia mutua con los estereotipos, estos mismos autores mencionan que no por eso tienen los mismos efectos sobre la discriminación, sino al contrario, en este aspecto son completamente independientes.

\section{Metodología}

Este trabajo se llevó a cabo bajo un enfoque cualitativo. Se utilizaron entrevistas en profundidad y cuestionarios para llegar a las vivencias, experiencias y perspectivas de los entrevistados y las entrevistadas. Para complementar los análisis derivados de las entrevistas en profundidad se tomará en cuenta también un análisis del lenguaje no verbal de las personas entrevistadas. Hay que especificar en este artículo científico que las entrevistas a los distintos actores se las hizo de manera aleatoria, contando con el apoyo del Instituto Nacional de Migración con sede en Juárez.

Para el análisis del lenguaje no verbal se tomarán en cuenta los 'canales del cuerpo' que “incluyen la apariencia general, la postura corporal, las gesticulaciones, el movimiento, el contacto físico, la distancia y la orientación” (Keats, 2009:67). 
Las entrevistas fueron realizadas a tres actores principales que giran en torno a las dinámicas de la migración; agentes migratorios de Estados Unidos, Polleros o coyotes y personas migrantes. Entendiendo que dichos actores participen directamente en el proceso migratorio. Tanto los polleros como los agentes migratorios, son actores con una enorme dificultad de hacer conexión ya que por cuestiones de alcance (capital económico y social) el acceso se limita. A su vez fue complicado ya que ambos actores sufren de riesgo identitario (el Pollero ya que su "negocio" es ilegal, por su parte el agente migratorio que siempre está temeroso o en alerta de un atentado terrorista).

Por tanto, se entrevistó solo a un pollero y a un agente migratorio, teniendo que realizar una serie de visitas, para lograr una entrevista completa y satisfactoria con cada uno de ellos. Conforme a los y las migrantes fue un proceso con más acceso, ya que lo único necesario fue solicitar un permiso que rápidamente fue otorgado por las autoridades pertinentes, así que tuvimos acceso a la casa del migrante ubicada en Ciudad Juárez donde se realizaron 11 entrevistas, se eligió dicho límite ya que las entrevistas y sus variables comenzaban a ser repetitivas o predecibles.

\section{Análisis de la información y resultados}

\section{Caso 2: "El tío" de Ana}

El tío de Ana (se le llamó así para proteger su identidad), tiene 49 años de edad, es padre de cuatro hijos, todos mayores de edad; vive en unión libre, señaló ser originario de Fresnillo, Zacatecas. Refiere que no es la primera vez que cruza a Estados Unidos, han sido unas tres ocasiones, la primera vez fue hace once años aproximadamente, en el año 2018. En aquella ocasión buscaron un "pollero" o "coyote", que les cobró 3,000 dólares, el pago fue por adelantado. Entraron por el Valle de Juárez, a través de los sembradíos, pasaron caminando y en la noche; su destino final era la Ciudad de Denver, Colorado.

Relata que la primera vez que lo hizo fue por conocer, las otras ocasiones fue por trabajo. En una ocasión, el pollero los llevó a un apartamento en El Paso, Texas, y los abandonó por una semana. No volvieron a saber de él. Tuvieron que contactar a otra persona para llegar a su destino, quien les cobró 1,700 dólares por llevarlos a la estación de autobuses. En su tercer intento, se escondieron en un vagón de tren que los iba a llevar a Denver, el tren nunca se movió, y fueron descubiertos por los oficiales de migración. En esta última ocasión refiere que eran como veinte 
personas, había mujeres y niños, a todos los detuvieron para ser deportados. Señaló que no recibió maltratos, aunque observó que otras personas sí eran maltratadas física y verbalmente. Finalizó su testimonio con la siguiente frase "ahora es más difícil, creo que ya no lo volvería a hacer, son experiencias muy difíciles, y sufres mucho".

\section{Caso 3: Charly, y la experiencia de la primera vez}

Charly, es un joven de veinte años de edad, soltero, sin hijos y originario de Ciudad Juárez, Chihuahua. La motivación de Charly fue de tipo afectiva, pues quería estar con su novia que vive en Fabens, una población cerca de la Ciudad de El Paso, Texas. Aclara que no fue por cuestiones de trabajo o porque tuviera necesidades laborales y económicas como la mayoría de los migrantes.

Esta fue la primera vez que Charly decidió cruzar con un "pollero"; en su testimonio señala que cruzó por Riberas del Bravo, el mismo día que se puso en contacto con el pollero ese mismo día cruzó. Sin embargo, estuvo escondido en una casa todo el día, y fue hasta la noche cuando cruzaron. El pollero los llevó por un camino de sembradíos, hasta llegar a la malla metálica que separa ambos países, por allí iban a cruzar, les dijo que una camioneta los esperaría del otro lado para llevarlos a su destino, primero les cobró y posteriormente los dejó solos. Les mintió.

El testimonio de Charly finaliza refiriendo que esta mala experiencia le cambió la vida, se arrepiente de haber cruzado así, y señala que "es mejor tramitar los documentos, y hacer bien las cosas", ahora vive con miedo de ser detenido por la policía.

\section{Agente 1}

El agente migratorio trabaja hace 40 años en la patrulla fronteriza y romper sueños y aspiraciones es parte de su trabajo, aunque eso no significa que sea su intención. Esta frase la comentó en una ocasión uno de sus compañeros, cuando vieron a un migrante pasar frente a su camioneta durante una de sus jornadas de trabajo.

Se convirtió en agente de migración porque es parte del servicio que presta a su país, porque le gusta el orden y el respeto a las leyes, pero esto no le impide ser un hombre sensible que justifica hasta cierto punto este cruce ilegal por la situación de crisis y pobreza que se vive en México. Justifica su labor, aunque otras personas lo consideran como una ocupación donde se hace una labor injusta, comenta que lo hace porque alguien la tiene que realizar y es una forma digna de ganarse la vida. El la realiza con el respeto que se merecen los demás individuos como seres 
humanos, comenta. El agente es un hombre de aproximadamente 55 años, nacido en Laredo, Texas, hijo de madre migrante y padre México-norteamericano. Fue educado en una familia religiosa con aprecio a los valores morales, la mayoría de ellos muy tradicionales y apegados a la cultura mexicana. Le enseñaron el respeto por el otro y por esta razón considera que hoy es un hombre de bien. En su casa se hablaba el inglés y el español de manera fluida y es por esta razón que conoce los dos idiomas. Recuerda que cuando era niño había un acontecimiento anual en su pueblo que difícilmente veremos ahora: un día libre en la frontera. Era una jornada especial que permitía a hombres y mujeres pasar del lado mexicano al lado norteamericano. Este era consentido por las autoridades norteamericanas y se tomaba como un día de convivencia entre aquellos(as) que no se veían.

\section{El tío mono (pollero)}

El tío mono es un hombre de 35 años de edad. Hace 10 años decidió dedicarse a ser pollero, para agarrar dinero fácil. Entre sus experiencias positivas menciona el agarrar dinero fácil y rápido, así como conservar amistades de personas a las que alguna vez ayudó a cruzar. Entre las negativas recuerda cuando un niño se les cayó al río y se enterró un palo teniendo que volver de emergencia para atenderlo, en esa ocasión refiere haber sentido miedo de que se le muriera el niño, más que preocupación porque los agarraran los agentes migratorios, desde entonces decidió no cruzar niños. Menciona que casi siempre era muy fácil cruzar "hasta que pusieron el muro", fue cuando decidió retirarse. Reconoce los riesgos que hay al intentar cruzar ilegalmente. Cree que en la sociedad se le da al pollero una imagen positiva pues recuerda se decía "este bato es fregón", además de que la misma gente lo promociona, y eso significa agarrar más dinero, sin embargo, nadie en su colonia sabe que a eso se dedicaba. La percepción que su familia tiene sobre lo que hacía es que más allá de ser algo ilegal, es una fuente de ingreso. Comentó que, si un migrante se accidenta, el atenderlo o abandonarlo varía según el pollero que esté a cargo del cruce, en su caso nunca los dejaba solos, incluso los entregaba hasta el domicilio al que le dijeran. Considera que hacía un servicio a quienes querían estar en Estados Unidos y no habían podido.

\section{Conclusión}

Con base en los resultados obtenidos en los análisis de las entrevistas realizadas, concluimos que las personas involucradas en este proceso migratorio en ningún momento dejan de ser susceptibles al estigma social, estereotipos y prejuicios que la población en general determina 
arbitrariamente hacia ellos y ellas. Sin embargo, la información recabada nos demuestra que a pesar de estos no todas las personas y no en todos los momentos cumplen con lo que demanda el estigma, el prejuicio y el estereotipo, ya que cada uno de ellos ha vivido experiencias que aparentemente le han marcado la vida y que por lo general se relacionan con el tener que demostrar una actitud empática hacia las demás personas que viven desde otro punto este mismo fenómeno de la migración.

Creemos que si los datos estadísticos nos siguen dando muestra de la relevancia actual que tiene la migración entre México y Estados Unidos, aquellas personas interesadas en regularla, deben comenzar a tomar en cuenta el daño que se le hace tanto a la sociedad en general como a los participantes específicos al fomentar que se creen tanto estigmas sociales como prejuicios en contra de estas personas, por lo que proponemos que a partir de aquí se tenga una visión más amplia y más humanitaria en torno a este tema, pues solo así podremos alcanzar a comprenderlo en su totalidad para después actuar de una manera favorable en todos los sentidos posibles, sin pasar por encima de los Derechos Humanos de nadie.

\section{Referencias Bibliográficas}

Abrams, F.S., (1991) Política estadounidense de inmigración: ¿Cuan estrecha es la puerta? En Hofstetter, R.R, (Autor), La política de inmigración de los Estados Unidos. Argentina, Ediciones Tres tiempos. (pp 203-215)

Burbano Alarcón, M. 2010. La “teoría mimética” de René Girard y su aporte para la comprensión de la migración. Universitas Philosophica. 27(55): 159-181

Castañeda, Alejandra. 2012. La violencia de la ley: la legislación migratoria y el proceso de deportación. En Migrantes, desplazados, braceros y deportados compilado por París Pombo, M.D. pp 297-331 México.

Cornier, William H. y Cornier, L. Sherilyn. 2000. Estratégias de entrevista para terapeutas.

Fernández Poncela, Ana María. 2009. La investigación social: caminos, recursos, acercamientos y consejos. México: Editorial trillas.

Kassin, Saul; Fein, Steven, y Rose Markus, Hazel. 2010. Psicología Social. Queretaro: Artgraph. 
Keats, Dphne M. 2009. Entrevista: Guía práctica para estudiantes y profesionales. México: McGraw-Hill.

López Sala, Ana María. 2005. Inmigrantes y estados: la respuesta política ante la cuestión migratoria. España: Anthropos.

Mazurek, H. 2009. Migraciones y dinámicas territoriales. En Migraciones contemporáneas Contribución al debate compilado por Salazar, C., pp. 11-33. Bolivia: Plural editores.

Padilla, Hector. 2012. ¿Repatriado? Una historia de vida y su contexto. En Migrantes, desplazados, braceros y deportados, compilado por París Pombo, M.D. pp 209-248 México.

Rodríguez Ramírez, H. 2007. La migración internacional en el noreste de México y sus efectos socioeconómico. En La migración a Estados Unidos y la frontera noreste de México compilado por Arzaluz Solano, S. pp 27-75 México: El colegio de la frontera norte, Miguel Ángel Purrúa...

Rosaldo Renato. Cultural citizenship, Inequality, and multiculturalism. In: FLORES William V, BENMAYOR Rina., editors. Latino cultural citizenship: claiming identity, space, and rights. Beacon Press; Boston: 1997.

Santa Ana Otto. Brown tide rising: metaphors of Latinos in contemporary American public discourse. University of Texas Press; Austin: 2002.

Secretaría de Relaciones Exteriores. 2004. Reporte ejecutivo

Singer Audrey, Massey Douglas S. The social process of undocumented border crossing. International Migration Review 1997 [PubMed]

Zolberg Aristide. A nation by design: immigration policy in the fashioning of America. Russell Sage Foundation; Nueva York: 2006. 